\title{
Multiple Depth DB Tables Indexing on the Sphere
}

\author{
Luciano Nicastro ${ }^{1}$ and Giorgio Calderone ${ }^{2}$ \\ ${ }^{1}$ Istituto Nazionale di Astrofisica, IASF Bologna, Via P. Gobetti 101, 40129 Bologna, Italy \\ ${ }^{2}$ Istituto Nazionale di Astrofisica, IASF Palermo, Via U. La Malfa 153, 90146 Palermo, Italy
}

Correspondence should be addressed to Luciano Nicastro, nicastro@iasfbo.inaf.it

Received 30 June 2009; Revised 13 October 2009; Accepted 12 January 2010

Academic Editor: Joshua S. Bloom

Copyright ( $\odot 2010$ L. Nicastro and G. Calderone. This is an open access article distributed under the Creative Commons Attribution License, which permits unrestricted use, distribution, and reproduction in any medium, provided the original work is properly cited.

\begin{abstract}
Any project dealing with large astronomical datasets should consider the use of a relational database server (RDBS). Queries requiring quick selections on sky regions, objects cross-matching and other high-level data investigations involving sky coordinates could be unfeasible if tables are missing an effective indexing scheme. In this paper we present the Dynamic Index Facility (DIF) software package. By using the HTM and HEALPix sky pixelization schema, it allows a very efficient indexing and management of spherical data stored into MySQL tables. Any table hosting spherical coordinates can be automatically managed by DIF using any number of sky resolutions at the same time. DIF comprises a set of facilities among which SQL callable functions to perform queries on circular and rectangular regions. Moreover, by removing the limitations and difficulties of 2-d data indexing, DIF allows the full exploitation of the RDBS capabilities. Performance tests on Giga-entries tables are reported together with some practical usage of the package.
\end{abstract}

\section{Introduction}

Astronomical projects need to manage data which are directly or indirectly related to sky coordinates. As larger and larger datasets are collected by detectors at all wavelengths, it is more and more crucial to efficiently manage those data to speed up and optimize their exploitation. Modern database servers (RDBS), being highly optimized for managing large amounts of data, are the best available choice for today's astronomical project. The SQL language used to access the data provides several high-level functionalities; still it could be not enough since some scientific, in particular, Astronomy specific capabilities and tools are missing. Speed and flexibility of a query are strongly dependent on the indexing used for the underlying DB table(s). Even simple and in principle little resource-demanding tasks can become highly inefficient if performed on poorly indexed archives. Typically DB servers offer efficient indexing of one or more 1-d data using the so-called B-tree structure. However data produced by an astronomical experiment are typically related to sky coordinates which span a $2-\mathrm{d}$ space. Although it is possible to index such data using one or two simultaneous 1-d indexes like RA and Dec, the queries performance will be very poor since the search criteria on a 2 -d space can be much more complex than on a union of two independent 1-d space. Indeed the only possible queries that take advantages of such indexes will involve range checking along the two coordinates like $\alpha_{1} \leq \alpha \leq \alpha_{2}$ and $\delta_{1} \leq \delta \leq \delta_{2}$. In some cases, the RDBS provides built-in capabilities to manage 2 - $\mathrm{d}$ coordinates into index files using the $R$-tree structure. This would allow search criteria like "Find all objects within 2 arcsec of a given location." However these functionalities are far from being a standardized feature of RDBS; furthermore, there is room for optimization and specialization for astronomical usage.

Fast data access is, for example, required by tasks like cross-matching between two full-sky optical or IR catalogs. Another example is the automatic fast astrometry of a field aimed at quickly identifying transient sources, for example, gamma-ray bursts optical transients. This is a typical task of robotic telescopes. The transient source coordinates can then be automatically communicated to larger instruments in order to perform a more detailed study through spectral or polarimetric observations. One more example is the quick production of the map of a sky region showing some physical or statistical property of the underlying DB entries. This should be possible by using a simple, SQL standard query. 
Web-based tools managing large-area surveys would greatly benefit from fast RDBS response time too.

Luckily, like the amount of data, also DB servers power and flexibility increase with time making possible to dream, actually to start thinking about a DB table with sub-arcsec sky resolution, that is, comparable to a CCD pixel. We will see that DIF is just ready for this! Building a Stellarium-like (http://www.stellarium.org/) or GoogleSky-like (http://www.google.com/sky/) tool to browse an astronomical data archive would then be the natural choice, with the obvious difference being that the user can retrieve or work interactively with scientifically meaningful data. Discussing the technical and financial problems of such an ambitious project is beyond the scope of this paper. We only mention the ESO VirGO project (http://archive.eso.org/cms/virgo/) as a possible initial pathfinder.

The open-source package DIF (Dynamic Indexing Facility) [1] is a combination of a C++ library, a Perl script, and SQL stored procedures allowing the automatic indexing of MySQL tables hosting sky coordinates. The approach adopted was to "discretize" a 2-d space and map it onto 1$\mathrm{d}$ space using a pixelization scheme. Each pixel is then tagged with a unique integer ID so that an index can be created using the standard B-tree. The usage of MySQL was a natural choice as it was the only open-source RDBS which allowed us to add a custom "DB engine" to the server. At the moment it remains the only RDBS supported by DIF.

\section{DIF Motivation and Architecture}

The motivations for an efficient management of DB tables hosting data with spherical coordinates are not different from those of any other type of data. Exploiting large datasets and performing complex types of queries are unfeasible, in a reasonable amount of time, without an effective indexing. Typically indexes are not just used to quickly select entries within a given table, but also to define a "relation" with the content of other tables in the DB. The capability to perform "joined" queries is a key feature of relational databases. As mentioned, indexing 2-d data (in order to allow spatial queries) is quite different from the $1-\mathrm{d}$ case because any "sequential" data ordering, for example, along one direction, inevitably causes data close in space to be distant in a table, or disk file. Consider, for example, a catalog of objects; in this case one can slice the sphere along the declination (latitude) axis and sort in right ascension (longitude) within each strip. Objects in each strip can be stored into a separate table. Let us now assume that, upon a user request, a dedicated task exists which identifies the stripes of interest. If only one strip is affected by the query, then, depending on how many objects are stored in that table/file, the task of identifying and delivering the requested data is relatively easy. In the case that several strips are to be searched, the task will simply access and search all the affected tables. Of course if the slicing is also performed along the RA axis, the amount of data per table reduces, as does the response time of such task. Moreover, instead of this geographic-like grid, one can adopt any kind of sky slicing and manage the requests accordingly. All this would work fairly well for a cone-search service. Actually this method is adopted by several catalog search tools (e.g., the VizieR service, vizier.ustrasbg.fr) with acceptable performance. Of course the search efficiency depends on the relative size of the queried region with respect to that of the slice. The closer the better. Also the average number of objects falling into a slice must be not too small nor too large. For example, the 1 billion objects USNOB1.0 catalog uses strips of $0 .{ }^{\circ} 1$ in declination, which means that an average of half-million objects is present in each strip.

But what about importing those tables into a RDBS to exploit its capabilities? For example, the usage of an RDBS would allow to easily perform spatial joins on various catalogs. Not to mention the fact that a standard language (SQL) is used to perform queries. This offers an infinitely greater flexibility compared to a custom program which performs a limited number of tasks, only works on a given catalog, requires maintenance, and probably has little portability. As already been said, once the data have been inserted into a DB, one has to find an efficient indexing method for 2-d data. A simple 1-d indexing on declination could be investigated in order to estimate the RDBS performance with respect to the previous custom management. We have not done so and we do not expect a significant leap in performance when compared to the custom case. In particular the RDBS optimizer would be unable to take advantage of the index to perform spatial joins. However other advantages of using an RDBS remain.

The next step is then to find a method to cover the sphere with cells (pixels) and number them. The resulting set of integers can then be indexed using the conventional Btree and therefore the whole potential of the RDBS can be exploited. So what we need is a 2-d to 1-d mapping function: each entry in the table will have one single integer ID and a sky area maps to a set of integers. A further step is identifying the most appropriate pixel size. Likely this is project dependent, but we will see that having some thousands entries per pixel could be a good choice. Among the various available functions, those implemented by the Hierarchical Triangular Mesh (HTM) [2] and the Hierarchical Equal Area isoLatitude Pixelization (HEALPix) [3] tessellation schema are the most suitable to our aim for a number of reasons; for example, (1) they cover the sky uniformly with no singularities at the poles, (2) the algorithm to compute the pixel IDs is very fast, (3) they implement, or allow to easily implement, tasks to work with the pixel IDs, (4) they are commonly used in Astronomy, and (5) they are available as opensource C++ libraries (see http://www.sdss.jhu.edu/htm/ and http://healpix.jpl.nasa.gov/). See Section 3 for more details.

The DIF core is represented by a MySQL DB engine implemented into a $\mathrm{C}++$ class. Functions to perform region selection (and other tasks) use the HTM and HEALPix libraries to calculate the IDs of the pixels included or touched by the requested region. From the user point of view a dynamic table is generated which contains only the entries falling in the region. A DIF-managed table can be queried as any DB table with the additional possibility to perform queries on circular and rectangular sky regions, and so forth (see below). In other words DIF extends the SQL language 


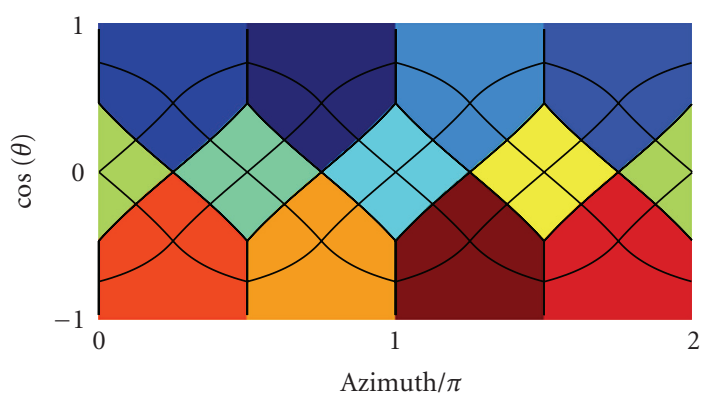

(a)

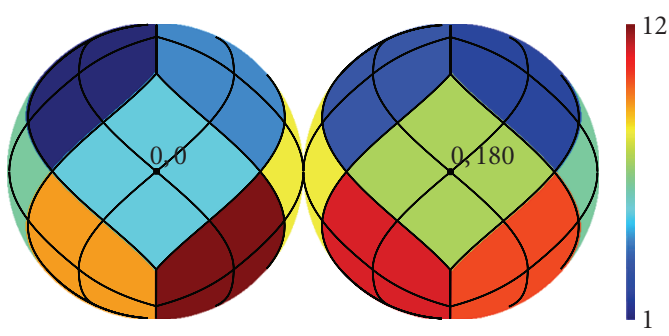

(b)

FIGURE 1: The 12 HEALPix base pixels color coded for the "ring" scheme. Overplotted the pixel boundaries for $k=1$ which gives 48 pixels.

and removes the need to write complex procedures or to use temporary tables to perform 2-d selections. Notably, the current version of the package has no limitations on the number and type of simultaneous pixelizations applicable to a table and the user can choose between two possible types of select queries on spatial regions: (1) give the region of interest in each query string, and (2) first define the region of interest, eventually selecting a specific pixelization depth, then perform any number and type of query on the DIF created view (see examples below). Which one is more convenient depends on the type of interaction of the DB client application and complexity of the query one needs to perform. What is important to note is that in the latter case the selected region is retained until a new region selection command is issued or the region selection is cleared. This property could be useful in the future to implement more complex selections involving union or intersection of regions.

Additionally DIF offers (a) useful User-Defined Functions (UDFs) mainly derived from the HTM and HEALPix libraries, (b) standalone programs to create fake test tables with spherical coordinates, and (c) HEALPix-specific IDL library which allows the user to obtain the result of a query as a sky map. So who should use it? Potential users are those wishing to manage in an automatic and effective way large datasets of astronomical data, of whichever kind: objects catalogs, observation logs, photon lists collected by spacebased high-energy detectors, single pixel counts of lowmedium resolution wide-field cameras, and so forth.

\section{HEALPix and HTM}

The HEALPix pixelization scheme [3] uses equal-area pseudo-square pixels, particularly suitable for the analysis of large-scale spatial structures. The base pixels are 12 with two different shapes: four identical ones are centered along the equator at $90^{\circ}$ step starting from $(0,0)$; the remaining eight, all identical, are centered at $45^{\circ}$ longitude offset and at a fixed poles distance of $z \equiv \cos \theta= \pm 2 / 3$ ( $\theta$ is the colatitude), four in the northern and four in the southern hemispheres (see Figure 1). The region in the range $-2 / 3<z<2 / 3$ is referred to as the equatorial zone, with the two remaining regions being the polar caps. Recursive subdivision of these pixels is performed keeping their centers equally distributed
TABLe 1: Relevant parameters for the HTM and HEALPix sphere pixelization.

\begin{tabular}{lcc}
\hline & HTM & HEALPix \\
\hline$N_{\text {pix }}^{\dagger}:$ & $8 \times 4^{d}$ & $12 \times N_{\text {side }}^{2}\left(\right.$ where $\left.N_{\text {side }}=2^{k}\right)$ \\
ID range: & {$\left[N_{\text {pix }}, 2 \times N_{\text {pix }}-1\right]$} & {$\left[0, N_{\text {pix }}-1\right]$} \\
Max $N_{\text {pix }}:$ & $\simeq 9.0 \times 10^{15}$ & $\simeq 3.5 \times 10^{18}$ \\
Max res.* $\left.{ }^{\prime \prime}{ }^{\prime \prime}\right):$ & $\simeq 1 \times 10^{-2}$ & $\simeq 4 \times 10^{-4}$ \\
& & $\left(\Omega_{\text {pix }}=\pi /\left(3 \times N_{\text {side }}^{2}\right)\right)$ \\
\hline
\end{tabular}

${ }^{\dagger} d$ (depth): $[0,25] ; k$ (order $\Leftrightarrow$ resolution parameter): $[0,29]$

* For HTM the maximum resolution is derived from the trixel minimum side, for HEALPix assuming a square-pixel equivalent area.

along rings of constant colatitude. Rings located in the equatorial zone are divided into the same number of pixels, the remaining rings contain a varying number of pixels. The two rings closest to the poles always have 4 pixels and going toward the equator the number of pixels increases by four at each step. The resolution of the HEALPix grid is parameterized by $N_{\text {side }}=2^{k}$, where $k$ assumes integer values being 0 for the base pixelization. It is called the "resolution parameter" or order. It is then $N_{\text {pix }}=12 \times N_{\text {side }}^{2}$. Table 1 shows the relevant pixelization parameters (see Górski et al. [3] for more details). The HEALPix library implements a recursive quad-tree pixel subdivision which is naturally nested. The resulting pixel numbering scheme is then referred as the nested scheme. Alternatively the ring scheme simply counts the pixels moving down from the north pole to the south pole along each isoLatitude ring (see Figures 1 and 2). The usage of the ring scheme is not recommended to index tables like objects catalogs which are typically queried on small sky regions. In fact in this case a data sorting will not result into an efficient "grouping" like that obtainable for the nested scheme where to "close-on-sky" pixels correspond "close-ondisk" data. This is important because data seek time is the main issue to face when very large tables are considered.

The C++ library released in HEALPix version 2.10 uses 64-bit long integers to store the pixels IDs. This allowed us to push the resolution of the pixelization to $\simeq 4 \times 10^{-4}$ arcsec. However, because of 64-bit floating point arithmetics limitations (e.g., minimum appreciable angular distance), this limit is not applicable for all the DIF implemented functions. 


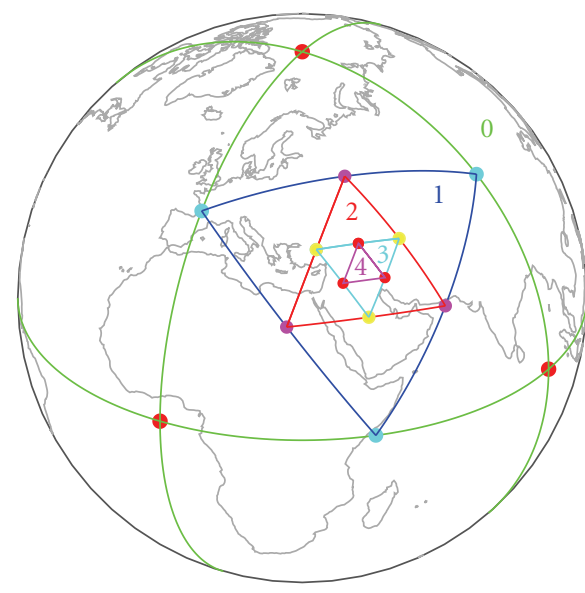

(a)

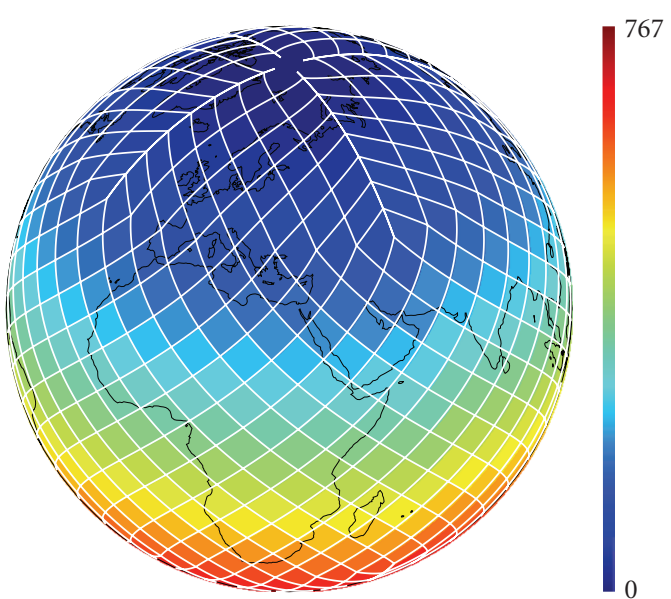

(b)

FIgure 2: (a) The 8 HTM base pixels and recursive subdivisions on Earth surface. The "depth" $d$ of the trixels is marked. (b) HEALPix tessellation at order $k=3$ giving 768 pixels whose colors encode their actual IDs in the "ring" scheme.

The HTM sphere pixelization scheme [2] uses triangular pixels which can recursively be subdivided into four pixels. The base pixels are 8, 4 for each hemisphere. They are obtained by the intersection on the sphere of 3 major big circles. On Earth they can be represented by the equator and two meridians passing at longitudes $0^{\circ}$ and $90^{\circ}$ (see Figure 2). These base spherical triangles all have the same area. Each of them can then be further divided into four spherical triangles, or trixels, by connecting the three sides middle points using great circle segments. As can be seen from Figure 2, from the first subdivision onward the resulting trixels are no longer equal area. The scatter of the trixels area remains within the $\pm 70 \%$ of the mean area, which is $2 \pi / 4^{d+1}$, with $d$ being the depth or level (step of recursive subdivision) of the trixel. For a given depth the number of trixels is $N_{\text {pix }}=8 \times 4^{d}$. The minimal side length is $\pi / 2^{d+1}$ and the maximal is $\simeq \pi / 2$ times the minimal length. The HTM indexing algorithm performs recursive subdivisions on a unit sphere using a quad-tree nested scheme and can identify each trixel both by integer and string IDs which refer to the vertices of the parent trixel counted counterclockwise (see Kunszt et al. [4] for more details). This algorithm has proven to be very efficient for selecting point sources in catalogs and was specifically developed for the Sloan Digital Sky Survey (see http://www.sdss.jhu.edu/ and [5]). Table 1 shows the relevant pixelization parameters. Note that, similarly to the HEALPix case, the depth 25 limit is imposed by the 64-bit floating point calculations. It can be lifted up to 30 if 128 bit variables and functions will be introduced in the source code.

Unless one needs to access a large fraction of a table and perform spatial analysis and map plot, we suggest to prefer the HTM pixelization because it offers a larger set of functions with respect to HEALPix. We will see below that the HEALPix IDs of any set of selected rows can still be computed using the DIF functions at query execution time.

\section{Using DIF}

Here we give a brief technical description of the DIF components and its capabilities. Installation instructions and other details can be found in the user manual distributed with the package and available at the MCS( My Customizable Server, ross.iasfbo.inaf.it/MCS/) website. The DIF source code can be downloaded from the same place as a compressed tar file. Note that being the DIF DB engine an extension to the MySQL server, it requires that MySQL was/is installed using the source package and that the path to the source code location is supplied in the configuration step. DIF adds the following components to a MYSQL server:

(1) A DIF database with the two tables: dif and tbl. The first is a dynamic table listing pixel IDs; the second retains all the information about the managed tables.

(2) A set of SQL stored functions useful to get information from the tbl table. Users can use them as an example to implement their own functions/procedures.

(3) A set of SQL callable C++ utility functions usable independently from the table type or query executed. Most of these external UDFs make use of the HTM and HEALPix libraries.

(4) The DIF DB engine, which feeds the DIF. dif table, and a set of DIF specific sky region selection functions. They are the compound of a $\mathrm{C}++$ class plus functions which rely on the HTM and HEALPix libraries to dynamically, and transparently, compute the pixel IDs of the region of interest.

(5) The dif Perl script used to perform various management tasks. It requires the DBD : : mysql Perl library to work (see Section 4.1).

(6) For each DIF indexed table. a number of views and triggers will be created. 
Note that DIF. dif is a table whose content is dynamically generated by the DIF DB engine each time it is accessed, transparently to the user, based on query search criteria. A simple "SELECT * from DIF.dif" without the usage of one of the DIF specific sky region/pixels selection functions will result in an "Empty set." The DIF.tbl table hosts all the necessary information about the DIF-managed tables, like pixelization schema, depth/order, and name of the fields with the coordinates. However, the only DIF components a user needs to know about are two: (1) the dif script (for a DB administrator) and (2) the DIF functions accessible within MySQL queries. They are listed in Table 2. A detailed description is reported in the user manual.

4.1. The dif Script. As mentioned, this section is of interest only for a DB administrator. All the various actions related to the management of a table via the DIF package are performed using a Perl script: dif. Its parameters and flags can be viewed typing dif --help. Its usage is straightforward and we give here a partial list of examples.

Let us assume that a DB MyDB with a table MyTab exists, that the sky coordinates of the entries are reported as centarcsec, and that the field names are RAcs and DECcs. To index the table with an HTM tessellation with depth $=8$, the command to give is

$$
\begin{aligned}
& \text { dif --index-htm MyDB MyTab } 8 \\
& \text { "RAcs/3.6e5" "DECcs/3.6e5", }
\end{aligned}
$$

and the result will be the following

(1) a column named htmID_8 of type MEDIUMINT UNSIGNED ( 3 bytes) and default value 0 will be added to the MyTab table,

(2) the column will be filled with the IDs of the trixel where that entry falls,

(3) an index is created on the htmID_8 column,

(4) a new entry is inserted into the table DIF.tbl with the values:

MyDB MyTab 108 RAcs/3.6e5 DECcs/3.6e5,

(5) a view named MyDB.MyTab_htm is created. It contains the appropriate INNER JOIN of the htmID_8 column with the dynamically populated column DIF. dif.id plus other statements (see the manual),

(6) a trigger named MyDB.difi_MyTab is created. It is used to automatically calculate the htmID_8 value when an INSERT query is executed.

Moreover, if two or more pixelization depths are to be applied to the table, they can be given simultaneously as a comma-separated string. For example, to apply depths 8 and 12 , the command would have been

$$
\begin{aligned}
& \text { dif --index-htm MyDB MyTab "8,12" } \\
& \text { "RAcs/3.6e5" "DECcs/3.6e5". }
\end{aligned}
$$

A spatial query on the MyTab_htm view performed via DIF_HTMCircle or DIF_HTMRect/HTMRectV will make use of all the available depths simultaneously by performing a recursive "erosion" of the requested region. Let us say that we have three indexes, 8,10 , and 12 , then the algorithm will find, in this order, that (1) the $d=8$ trixels fully contained in the region, (2) the $d=10$ fully contained trixels in the remaining area, and (3) the $d=12$ fully and partially contained trixels in what is left of the region. It could happen that no $d=8$ or/and $d=10$ trixel is found.

Sometimes it could be convenient to use only one of these pixelizations, for example, when the queried region size is close to the trixel size of one of the available depths. In this case the user can issue the command

$$
\text { dif --single-index-views MyDB MyTab }
$$

which will create views like MyDB.MyTab_htm_8 suitable to directly perform queries using one single pixelization depth. Of course this makes sense only if more than one pixelization was applied (see below how to perform sky regions SELECT queries on these views). To use a HEALPix tessellation with "nested" scheme, $k=8$, the command would have been

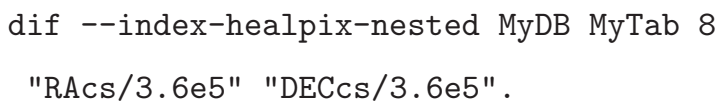

The added column name will now be healpID_1_8 (healpID_0_8 if the "ring" scheme was used) and the view will be MyTab_healp_nest. To remove an index and the related DIF facilities from a table, use a command like this the following:

$$
\text { dif --drop-index-htm MyDB MyTab } 8
$$

which refers to the first of the two examples above. If one wishes just to remove all the views and triggers from a table but keeping indexes and columns created with a dif command, then simply he/she can use dif --drop-views MyDB MyTab.

4.2. UDFs: SQL Routines and SQL Callable C++ Utilities. SQL stored functions are linked to the DIF database and give access to the information stored in the DIF. tbl table. They are usable in any query and are accessible to all MySQL users. For example, to find out which are the table columns used to get sky coordinates (in degrees!), one can execute the query

$$
\begin{aligned}
& \text { SELECT DIF.getRa ("MyDB", "MyTab"), } \\
& \text { DIF.getDec("MyDB", "MyTab"); }
\end{aligned}
$$

SQL callable functions are MySQL "external" function; still they can be used in any SELECT statement exactly like any DB server built-in function. Apart from the spherical distance calculation performed by Sphedist, they make available to the user HTM and HEALPix related functions. Note that the parameters "RA" and "Dec" are constants or the table column names corresponding to spherical coordinates. As mentioned, they must be in degrees; therefore conversion factors can be present, for example, RAcs/3.6e5. For example, to get the HTM IDs at $d=6$ of the neighbors of the pixel where the point $\left(100^{\circ},-20^{\circ}\right)$ falls, the command "select 
TABLE 2: DIF functions available in MySQL.

\begin{tabular}{|c|c|}
\hline SQL stored routines & SQL callable $C++$ utilities \\
\hline DIF.getHTMDepth (db, tab) & HTMLookup ( $d, \mathrm{Ra}, \mathrm{Dec})$ \\
\hline DIF.getHEALPOrder (db, tab) & HEALPLookup $(s, k, \mathrm{Ra}, \mathrm{Dec})$ \\
\hline DIF.getHEALPNested ( $d b, t a b, k)$ & HTMBary $(d$, ID $)$ \\
\hline DIF.getRa (db, tab) & HTMBaryC ( $d, \mathrm{Ra}, \mathrm{Dec})$ \\
\hline DIF.getDec (db, tab) & HTMBaryDist ( $d$, ID, Ra, Dec) \\
\hline Sky region/pixels selection & HTMNeighb $(d$, ID $)$ \\
\hline DIF_HTMCircle (Ra, Dec, $r$ ) & HTMNeighbC ( $d, \mathrm{Ra}, \mathrm{Dec})$ \\
\hline DIF_HTMRect (Ra, Dec, S1, [S2] ) & HEALPBary $(s, k$, ID $)$ \\
\hline DIF_HTMRectV (Ra1, Dec1, Ra2, Dec2) & HEALPBaryC (s, $k, \mathrm{Ra}, \mathrm{Dec})$ \\
\hline DIF_HEALPCircle (Ra, Dec, $r)$ & HEALPBaryDist $(s, k$, ID, Ra, Dec) \\
\hline DIF_HTMNeighbC (Ra, Dec) & HEALPNeighb $(s, k$, ID $)$ \\
\hline DIF_HEALPNeighbC $(\mathrm{Ra}, \mathrm{Dec})$ & HEALPNeighbC $(s, k, \mathrm{Ra}, \mathrm{Dec})$ \\
\hline DIF_setHTMDepth $(d)$ & Sphedist (Ra1, Dec1, Ra2, Dec2) \\
\hline DIF_setHEALPOrder $(s, k)$ & Auxiliary functions \\
\hline DIF_Sphedist (Ra1, Dec1, Ra2, Dec2) & DIF_useParam ( $d$ or $k)$ \\
\hline \multirow[t]{2}{*}{ DIF_FineSearch (var) } & DIF_cpuTime（） \\
\hline & DIF_clear () \\
\hline
\end{tabular}

Note: in DIF_HTMRect if $S 2$ is omitted it is assumed $=S 1$, that is, query a square; $s$ is the HEALPix schema switch: 0 for ring, 1 for nested.

HTMNeighbC ( $6,100,-20)$ " will return the string:

$37810,37780,37781,37783,37786,37788$,

37808 , 37809, 37811, 37817, 37821, 37822,

37823.

The first of the 13 IDs is always the one containing the input coordinates. Similarly to get the IDs of the neighbors starting from a given pixel ID, use "selectHTMNeighb(6, 37810)," which gives the same string above excluding the first ID, that is, 12 IDs. Note that for HEALPix the number of neighbors is not constant! More utility functions will be added in the future.

4.3. The DIF Specific Region/Pixels Selection Functions. These functions are those that allow the user to select the desired subset of table rows making a (transparent) use of the columns with the HTM and HEALPix IDs. They must be used either in the query WHERE clause or as the only argument of a SELECT statement to initialize the DB engine (see below). The pixel neighbors and region selection functions (the DIF_HTM... and DIF_HEALP..., see Table 2) only give results if applied to the corresponding HTM or HEALPix views as they produce a list of IDs in the dynamic table DIF.dif, which is then used as a reference table to join the managed table. For these functions, "RA" and "Dec" are the table column names corresponding to the sky coordinates. Also in this case, to have them in degrees, conversion factors can be present. Note how these functions do not require the pixelization parameters as they are directly managed by the views they apply to. For example, DIF_HEALPNeighbC only requires the coordinates column names, compared to the UDF HEALPNeighbC which also requires scheme ID and order $k$. This dynamic management of the table is a specific capability of the DIF DB engine. Some query examples with direct region selection are

$$
\begin{gathered}
\text { SELECT } * \text { FROM MyTab_htm WHERE } \\
\text { DIF_HTMCircle }(33,44,30) ; \\
\text { SELECT } * \text { FROM MyTab_healp_nest WHERE } \\
\text { DIF_HEALPCircle }(33,44,30) ; \\
\text { SELECT } * \text { FROM MyTab_htm_8 WHERE } \\
\text { DIF_HTMRect }(33,44,40) ;
\end{gathered}
$$

The first two queries return all the entries in a circular region centered at $\alpha=33^{\circ}$ and $\delta=44^{\circ}$ with radius $30^{\prime}$. The third will select entries in a pseudo-square region with same center and sides length of $40^{\prime}$ along RA and Dec.

Alternatively one can initialize the region of interest once and then execute any number of queries on the resulting entries. If multiple indexing has been applied (either HTM or HEALPix), then one can also choose to use only one depth/order, for example, $d, k=8$. In this case the function DIF_useParam must be used. A typical sequence of queries, for example, on an optical sources catalog, is:

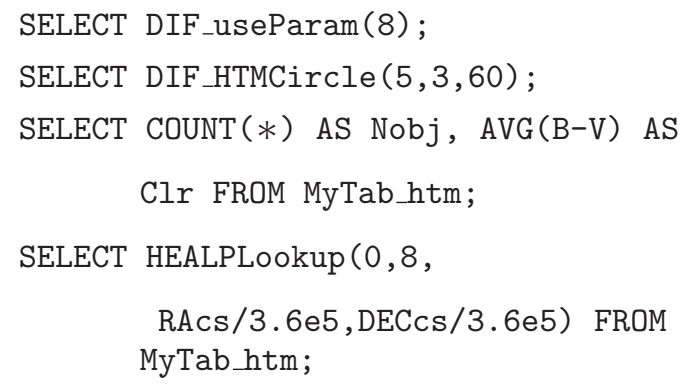

The first two SELECT just set DIF internal parameters returning 1 on success. The next query shows number and 
average "color" of the sources within $1^{\circ}$ around $\alpha=5^{\circ}, \delta=$ $3^{\circ}$. The last query will calculate and return the HEALPix IDs (order 8, ring scheme) of these sources. Note that cent-arcsec coordinates are assumed. Omitting to use DIF_useParam or giving it the value 0 would instruct DIF to use all the available depths (or orders) to perform the selection. This is the default. Also note that the region selection query can be followed by any number of queries on any table view. Only the rows falling into the selected region will be affected by these queries until a new region selection command is issued. A DIF_clear() can be issued to reset all the internal parameters. The MySQL server is a multithread process which means that it creates a new work environment for each client connection. DIF functions, being part of the server, set thread-specific parameters like any other MySQL intrinsic function. Their status and values are retained until the connection is closed. When a new connection with the server is established, the initial status of a DIF parameter is unpredictable (like the value of an uninitialized variable in a program).

An example which makes use of all the three described functions types is:

$$
\begin{aligned}
& \text { SELECT DIF.getRa("MyDB", "MyTab") as } \\
& \text { RAdeg, } \\
& \text { DIF.getDec("MyDB", "MyTab") as } \\
& \text { DECdeg, } \\
& \text { HTMNeighbC (6, RAdeg, DECdeg) } \\
& \text { FROM MyDB.MyTab_htm_6 WHERE } \\
& \text { DIF_HTMCircle }(10,20,5) \text {; }
\end{aligned}
$$

The result will be the list of neighbors for each object ID in the given circular region. Of course if more than one object falls in one pixel, the output will present several identical rows. It is trivial to modify the query to avoid this.

What about alternatives to DIF and in particular to the DIF DB engine and related UDFs? One could, for example, write a stored procedure which performs a cone search. First of all the table must have been created with a column suitable to receive, for example, HTM IDs at a given depth. For a given cone, one needs to write a function to calculate the IDs of the fully and partially covered trixels, then search the table for all the entries with these IDs excluding those falling outside the cone. The usage of a temporary table to store the IDs is unavoidable. This could pose efficiency problems but it is affordable. The usage of views and/or tables join would certainly help. Additionally one has to manually manage the insertion of new entries to calculate their IDs. The usage of triggers could help. We can continue with the reasoning requiring further facilities and tools and we will likely end up with a system with an architecture which resembles the DIF one, but is lacking the efficiency and flexibility intrinsic to DIF.

\section{Benchmarks}

In order to perform benchmarks on access time of DIFmanaged tables, we used several tables with fake entries. Some of them were produced generating fully random
TABle 3: Parameters for the HTM and HEALPix pixelization used in the tests.

\begin{tabular}{lcccc}
\hline$d / k$ & $N_{\text {pix }}$ & $\begin{array}{c}<\text { Area }> \\
\left(\operatorname{arcmin}^{2}\right)\end{array}$ & Rows/Pix. & Bytes* \\
\hline \multicolumn{5}{c}{ HTM } \\
\hline 6 & 32,768 & $1.26^{\dagger}$ & 89,290 & 2 \\
8 & 524,288 & 283 & 5,580 & 3 \\
10 & $8,388,608$ & 18 & 348 & 3 \\
12 & $134,217,728$ & 1 & 21 & 4 \\
\hline \multicolumn{5}{c}{ HEALPix } \\
\hline 6 & 49,152 & $0.84^{\dagger}$ & 59,526 & 2 \\
8 & 786,432 & 189 & 3,720 & 3 \\
10 & $12,582,912$ & 12 & 232 & 3 \\
12 & $201,326,592$ & 0.7 & 14 & 4 \\
\hline
\end{tabular}

$\dagger\left(\operatorname{deg}^{2}\right)$; *additional disk storage per row needed.

coordinates; this means that the sky distance between two sequentially generated entries could be anything in the range $\left[0^{\circ}, 360^{\circ}\right.$ [. It also means that a set of "close-on-sky" entries could be spread all over the disk data file. Other tables had their entries generated following a $\sim 1^{\circ}$ sky pixelization schema. This means that the data spread within the disk files is reduced by a factor equal to the number of pixels used to split the sky. For simplicity and analogy with the GSC 2.3 catalog (see below) we generated the entries over 32768 pixels corresponding to the HTM of depth 6 grid. Tables with average objects/pixel density in the range $\sim 10000-90000$ were produced (i.e., containing $0.4-3$ billion entries). We also produced and performed tests on "pixelized" and RA sorted (within each pixel) table. The 3 billion entries tables gave disk files size of $\sim 120$ GBytes. All the tables were created using the MyISAM DB engine. We avoided to split tables, and then the data files, by using the MERGE DB engine or the PARTITION BY option as, apart from management convenience, we do not expect any significant performance improvement on machines not equipped with a large number of disks. Region selections were performed using coordinates in degrees and cent-arcsec. Because the HEALPix library does not allow rectangular selections, for homogeneity reasons the selections were only performed on circular regions (also referred to as "disc" or "cone"). Four different pixelization depths/orders were used both for HTM and HEALPix (nested scheme) and the queries involved only one of them at the time. The results of benchmarks using the multidepth facility on very large tables will be reported elsewhere. The relevant parameters are reported in Table 3. We used a custom program to monitor the system resources usage by the select queries on DIF-managed tables. As expected, the most important hardware component determining the overall query execution time is the hard disk, in particular its data seek and access time. The higher the data contiguity the faster the query execution time. The used query implied calculations on the rows content; this is in order to force the load of the data into memory and not to have just a row counting. For each pixelization 

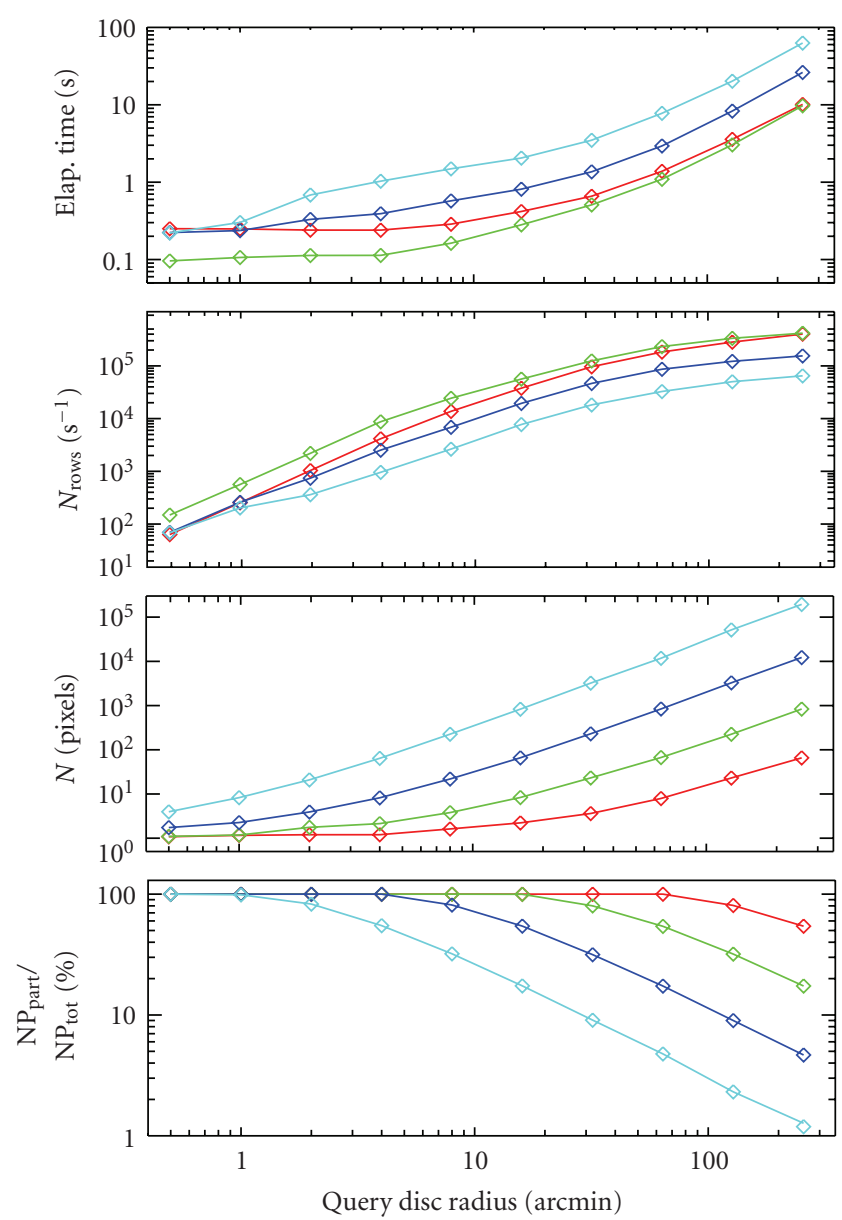

$\neg d=6(89290 \mathrm{objs} / \mathrm{pix}) \quad \diamond \quad d=10(348 \mathrm{objs} / \mathrm{pix})$

$\neg d=8(5580 \mathrm{objs} / \mathrm{pix}) \quad \diamond d=12(21 \mathrm{objs} / \mathrm{pix})$

(a)
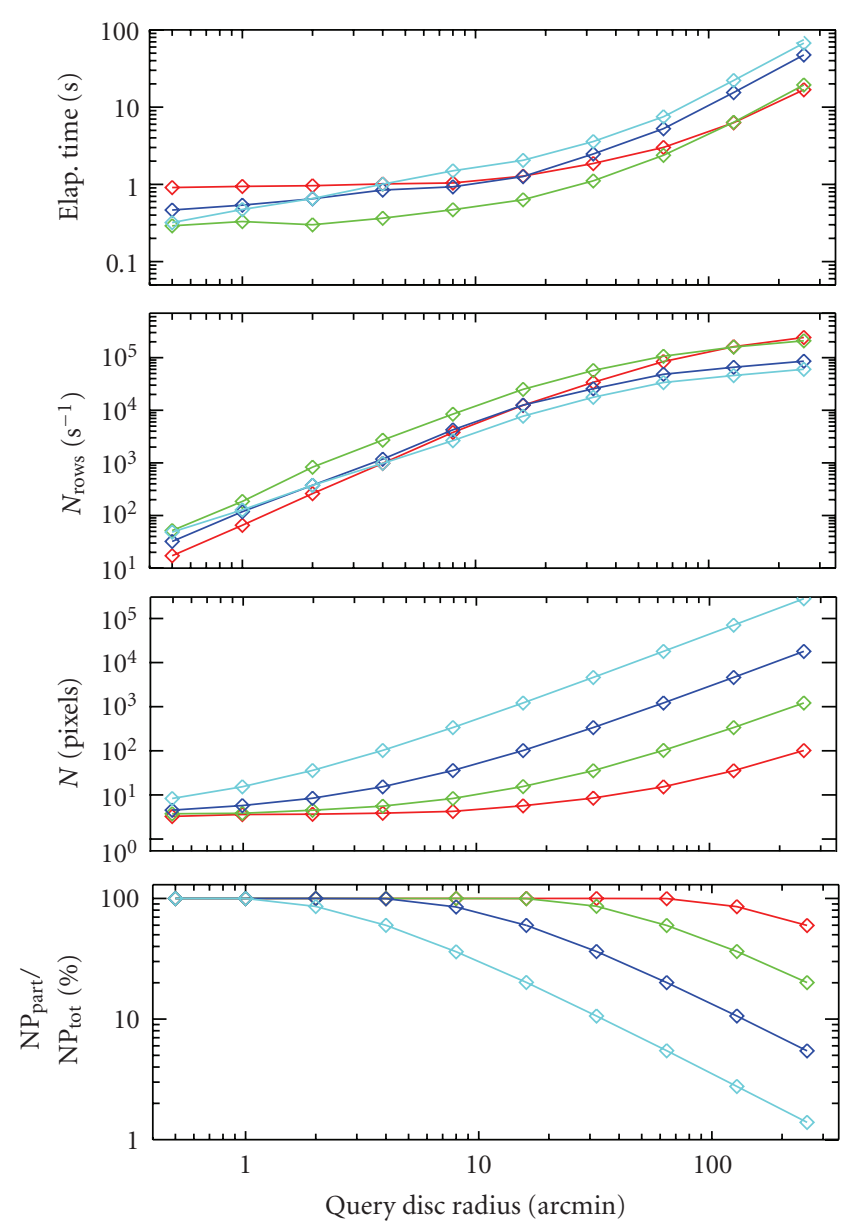

$\diamond k=6(59526$ objs/pix $) \triangleleft-\quad k=10$ (232 objs/pix)

$\diamond k=8(3720 \mathrm{objs} / \mathrm{pix}) \quad \diamond \quad k=12(14 \mathrm{objs} / \mathrm{pix})$

(b)

FIGURE 3: Select query execution times and other parameters for a DIF-managed table with $\sim 3$ billion entries as a function of the disc radius. Results for four different pixel scales are reported both for HTM (a) and HEALPix (b). Each point represents the average of the results from 50 queries performed on random sky positions.

Figure 3 shows, in a log-log scale, respect to the queried disc radius (top to bottom): (1) query execution times, (2) number of processed rows per second, (3) number of pixels involved, and (4) number of partially covered pixels respect to the total at a given depth/order. The used machine main characteristics are OS: Linux kernel 2.6.22.19 SMP x86_64, CPU: bi-proc. dual-core Intel Xeon 5130 @ 2GHz, RAM: 8 GBytes DDR2-667 $166 \mathrm{MHz}$, and Disk: RAID 1 on a 3 Ware Raid 9550SX-8 LP controller with two SATA2, 500 GBytes, $7200 \mathrm{rpm}, 3 \mathrm{Gbits} / \mathrm{s}$, reiserfs. The MySQL version used is 5.1.32 with key_buffer $=384 \mathrm{M}$, myisam_sort_buffer size $=64 \mathrm{M}$, query_cache_size $=32 \mathrm{M}$. The main results are as follows.

(a) For the fully random tables, query execution times are quite long because to fast index lookups corresponds slow disk file seeking (not scanning) which could affect the entire data table. Times grow in a quasi-linear way with respect to the region size and it is of $\sim 100$ rows s$s^{-1}$. This makes clear that, even though it is better than having, for example, a simple declination indexing, the usage of DIF on such sort of table is of very limited help. On the other hand, any real data would almost never be produced in a fully random way, but rather in a sequential way over sky "spots" or "stripes". This is, for example, the case of all-sky surveys performed in point-and-stare or slewing mode. A possible solution is to perform data clustering by sorting over the DIF index with lower depth/order using my isamchk -R index (see myisamchk manual).

(b) The HTM depth 6 sorted tables behave quite differently and show better performance when pixels with area of $\sim 200-300 \mathrm{arcmin}^{2}$ (HTM depth $=8$ and HEALPix order $=8$ ) are used to index the tables. This is particularly true for the table with $\sim 3$ billion rows we used, where we have some thousands entries per pixel (see Figure 3). Having a look to the curves measuring query execution time and number of processed rows per second as a function of the region size, we 


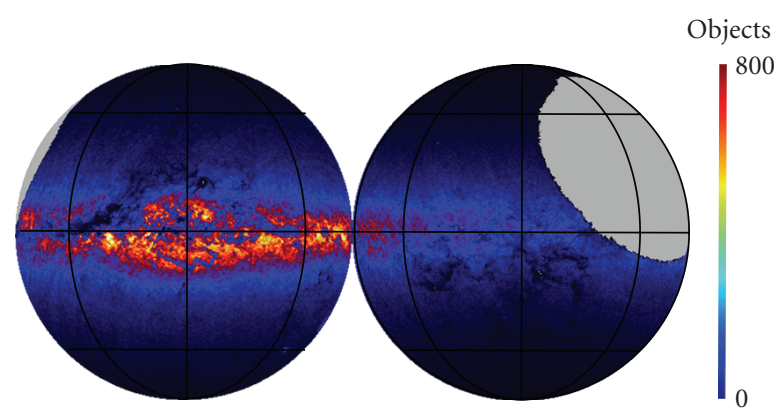

(a)

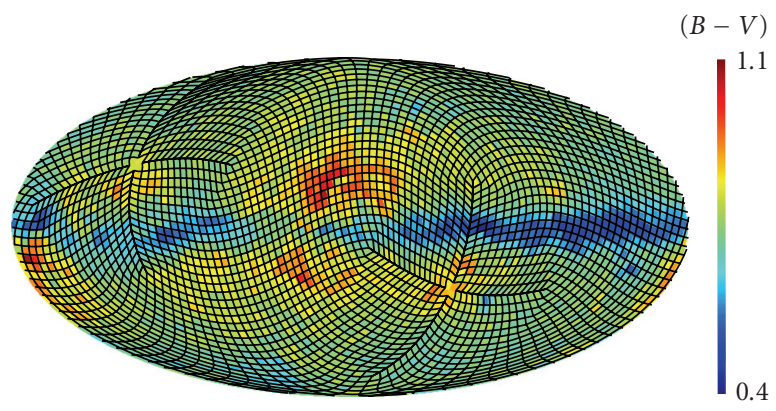

(b)

FIGURE 4: UCAC 2.0 full sky objects density map (a) and ASCC 2.5 objects average $B-V$ (b).

note that the behavior is similar for all the depths. However the point where the bending becomes more significant is depth dependent being shifted toward larger number of rows for higher depth pixelizations. As a consequence of this, we also note that, when some $1 \times 10^{5}$ rows are involved, $d=6$ starts to perform like $d=8$. The rows/s processing limit is $\sim 5 \times 10^{5}$. For tables with $\sim 1$ billion rows or less we found that higher resolution pixelizations $(d, k=10,12)$ perform not too differently from the $d, k=8$ case. The reasons for this are not so simple to investigate considering the many parameters involved. We note that for a given region size, when the pixel size reduces, the number of joins on pixel IDs in the query increases but the number of entries falling into partially covered pixels, for which a direct distance calculation from the center of the circle is required, decreases. It is likely that, depending on DB server and machine characteristics, the performance can vary with pixel density and number of join operations required. However, as already been mentioned, we do not note any significant dependence of the query execution time on the total CPU usage time, which is negligible. So, once again, the attention should fall on the data seek/access time and consequently its impact for the various pixel densities. Let us assume that a given circular region only partially covers 1 trixel at $d=8$; this is typically the case for $\mathrm{a} \sim 1^{\prime}$ radius region. In this trixel entries coordinates are randomly distributed. However we will access all of them on disk, so, in some way, they are accessed quite efficiently having a "contiguity factor" $f_{c}=$ $1 / 16$ (being the data clustered on $d=6$ trixels and for each depth step there is a factor four increase in the number of trixels). It is not a constant, but let us say that at $d=10$ the very same region partially covers two subtrixels of that trixel. The number of entries to seek/access reduces by a factor eight. Considering that the various processes involved will likely put in cache memory adjacent data, we can assume that there is one single data seek operation to access these rows. However now it is $f_{c}=1 / 256$, that is, the data are a factor eight more fragmented. Now if we half the entries density, the benefit of the reduced seek time is a factor eight greater for $d=10$ respect to $d=8$. This explanation seems to us sufficient to account for the measured query performance as a function of pixel density and size; still other possibilities could be investigated. (c) Adding (in our case) the RAcs column to a given table index and sorting the data on disk with this new combined index reduce the query execution time by a variable, still not very significant amount of time. On the other hand, time reduces by up to an order of magnitude if one uses directly in the WHERE clause of the query the RAcs column to delimit a range. This is what we did in the customized catalogs used for astrometry and objects matching in the REM [6] produced images (see below). Moreover, if one makes use of the buffering capabilities of the MySQL server by performing more than one query on the same sky region, the queries other than the first one will be executed in a very fast way. This strategy can easily be implemented in robotic telescopes software, for example, for GRB alerts!

(d) The usage of the "ring" scheme for HEALPix indexed tables is not recommended because any table sorting which makes use of such index will give for adjacent on sky pixels not adjacent position on disk. This effect is less relevant in the extreme cases when a few or the majority of the pixels are interested by a selection. Because of the poorer performance measured and because the calculation of the HEALPix IDs of table entries can be computed at query execution time, for a general purpose table we suggest to use always the HTM indexing.

\section{MCS-IDL Contributed Library}

MCS offers a client interface to the IDL language. We have implemented a library useful to interact in a very simple way with the MySQL server from this language. A further library useful to plot data on the sphere using the HEALPix IDL library is available too. DIF-managed tables data are easily plottable using this library. It is enough to put them on the user's IDL path together with the HEALPix library. These libraries are retrievable from the web. Here we show their usage on customized optical and IR catalogs.

6.1. Optical and IR Catalogs Sky Maps. For our tests on real data we used catalogs which are routinely used for realtime objects identification in images taken by the IR/optical robotic telescope REM (see Molinari et al. [6]). Among them are GSC 2.3, GSC 2.2 ( 18th mag threshold), USNO B1.0, 
2MASS, UCAC 2.0, and ASCC 2.5 (see the websites: wwwgsss.stsci.edu/, www.nofs.navy.mil/, www.ipac.caltech.edu/, ad.usno.navy.mil/, cdsarc.u-strasbg.fr/). Using DIF, we have indexed these catalogs with various pixelizations. The performed tests confirm the results obtained for the fake tables, that is, the fastest access time is achieved by using pixels of size $\sim 1^{\circ}$. We recall that the GSC 2.3 is distributed as 32768 FITS files, each covering a $d=6$ HTM trixel, whereas USNO B1.0 and 2MASS are split in files covering $0^{\circ} .1$ in Dec, RA ordered in the slice. To further speed up the region lookup, which for REM is $10^{\prime} \times 10^{\prime}$, we added coordinates indexing. For optimization and disk space-saving reasons, coordinates were converted into cent-arcsec and therefore packed into 4byte integers. Then a "unique" combined index was created on HTM ID plus coordinates. As already noticed in [1], with such an index and the usage of coordinate ranges in the WHERE clause to further reduce the number of rows to seek/access, queries on regions of size $10^{\prime}-30^{\prime}$ take $\sim 20 \mathrm{~ms}$.

Using IDL routines to visualize as sky maps data read from MySQL tables is quite straightforward. As said, the HEALPix pixelization schema and routines can be used to this aim. For example, in Figure 4 we show the UCAC 2.0 sky objects density in galactic orthographic projection. The map was built using $k=8$, that is, a pixel size of $\simeq 14^{\prime}$. The sky region not covered by the catalog is shown in grey. In the same figure the average $B-V$ of the ASCC 2.5 objects in pixels of area $13.4 \mathrm{deg}^{2}$ is shown in galactic mollview projection. A simple (and basic) IDL program to produce the first map is:

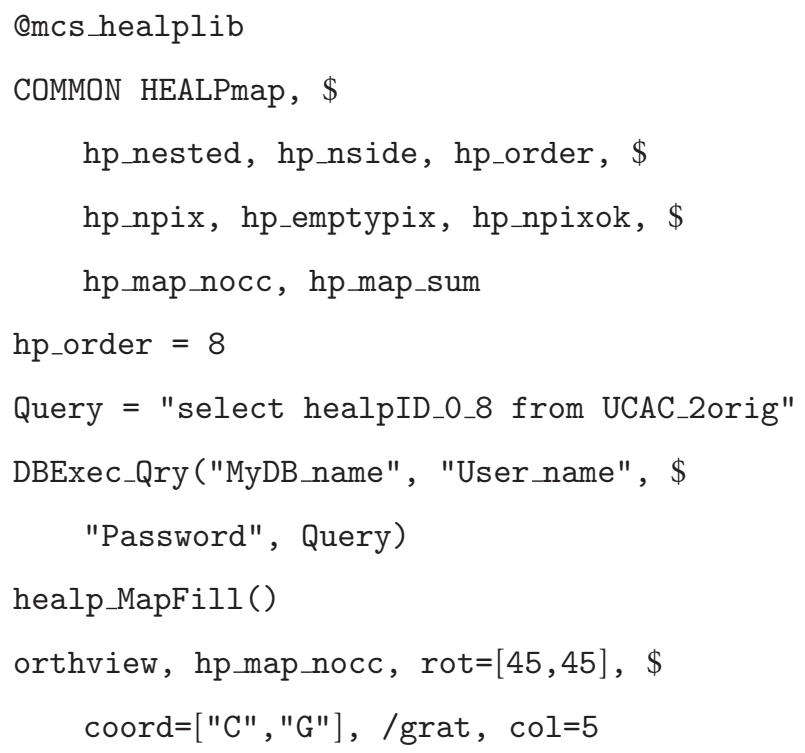

Note: (1) mcs_healplib (.pro) has all the high-level routines needed to build and save HEALPix maps; it also loads mcs_usrlib which is used to access MySQL tables. (2) The common block HEALPmap is used to pass the map parameters among the various routines. (3) If the UCAC_2orig table does not have the healpID_0_8 column (e.g., it is not indexed), then simply change the query into "select HEALPLookup (0,8, RAcs/3.6e5,DECcs/3.6e5) from UCAC_2orig" which will calculate the IDs for $k=8$ on the fly. (4) healp_MapSave () can be used to write the map into an FITS file. On the MCS website some demo programs are available to help write custom programs and routines.

\section{Conclusions}

We presented the DIF software package, its capabilities, and the results of benchmarks over very large DB tables. We have shown that for tables with billion entries a sky pixelization with pixel size of the order of $\sim 15^{\prime}$ (HTM $d=8$ ) gives best performance for select queries on regions of size up to several degrees. At this spatial resolution some thousands of entries fall in each of the $\sim 1 / 2$ million pixels; these entries have the same ID assigned in the DB table. We stress the fact that these results apply only in case some sky coordinates ordering is applied to the disk data file. If not, the disk seek time becomes so dominant to make the use of DIF, or any other indexing approach, meaningless. We also note that, in all cases, the CPU time accounts for only a few percent of the total query execution time; therefore great care must be put in choosing and designing the data storage. We have also shown the usage of HEALPix IDL libraries allowing the visualization and saving of sky maps obtained executing SQL queries directly from IDL.

The package is distributed under the GNU GPL and we encourage everybody to use it for their astronomical projects, in particular to manage object catalogs and large archives of data of any sort. We believe that the Virtual Observatory could certainly benefit by adopting a DIF-like approach and we hope that VO software developers will consider it. It is worth to note that not only Astronomy can benefit of the usage of DIF. It can be used by any project dealing with spherical data. Research fields like geology, geodesy, climatology, oceanography, and so forth all deal with geographical data. Those data can be stored into a DB, indexed on coordinates using DIF, and then efficiently retrieved, visualized, and used for a variety of purposes. Some new type of automatic navigation system having a DBS behind could also find more convenient a DIF indexing approach.

More capabilities and improvements are under development among which are cross-match UDF for automatic matching of objects in a circular or rectangular region selected from two different tables and the full porting to Mac OS. Other types of selection region, like regular and irregular polygons, rings, and so forth, or the adoption of commonly used region definition strings will be considered too. Though widely tested, the package is periodically update to remove bugs, improve performance, add new facilities, make it compatible with new MySQL versions, and so forth. Any comment or feedback is welcome. Users willing to contribute to the package development or testing are kindly asked to contact us. Please visit the website: ross.iasfbo.inaf.it/MCS/.

The now available DIF version 0.5.2 introduced some changes with respect to the version used when writing this paper. The reader is asked to read the user manual available with the software package at the mentioned website.

\section{Acknowledgments}

The authors acknowledge support from the astrometry group of the Turin Astronomical Observatory to have given 
us access to the various optical and IR catalogs. DIF makes use of the HEALPix [3] and HTM [2] packages. They also thank the referee for his/her helpful comments.

\section{References}

[1] L. Nicastro and G. Calderone, "Indexing astronomical database tables using HTM and HEALPix," Astronomical Society of the Pacific Conference Series, vol. 394, pp. 487-490, 2008, Edited by R. W. Argyle, P. S. Bunclark, and J. R. Lewis.

[2] P. Z. Kunszt, A. S. Szalay, and A. R. Thakar, "The hierarchical triangular mesh," in Proceedings of the MPA/ESO/MPE Workshop-Mining the Sky, A. J. Banday, S. Zaroubi, and M. Bartelmann, Eds., pp. 631-637, Springer, Garching, Germany, 2001.

[3] M. Górski, E. Hivon, A. J. Banday, et al., "HEALPix: a framework for high-resolution discretization and fast analysis of data distributed on the sphere," The Astrophysical Journal, vol. 622, no. 2, pp. 759-771, 2005.

[4] P. Z. Kunszt, A. S. Szalay, I. Csabai, and A. R. Thakar, "The indexing of the SDSS science archive," Astronomical Society of the Pacific Conference Series, vol. 216, pp. 141-144, 2000, Edited by N. Manset, C. Veillet, and D. Crabtree.

[5] J. Gray, A. S. Szalay, A. Thakar, et al., "Data mining the SDSS SkyServer database," in Proceedings of the 4th International Workshop on Distributed Data and Structures (WDAS '02), W. Litwin and G. Levy, Eds., pp. 189-210, Carleton Scientific, Paris, France, 2002.

[6] E. Molinari, S. Covino, S. D’Alessio, et al., "REM, automatic for the people," Advances in Astronomy. In press. 

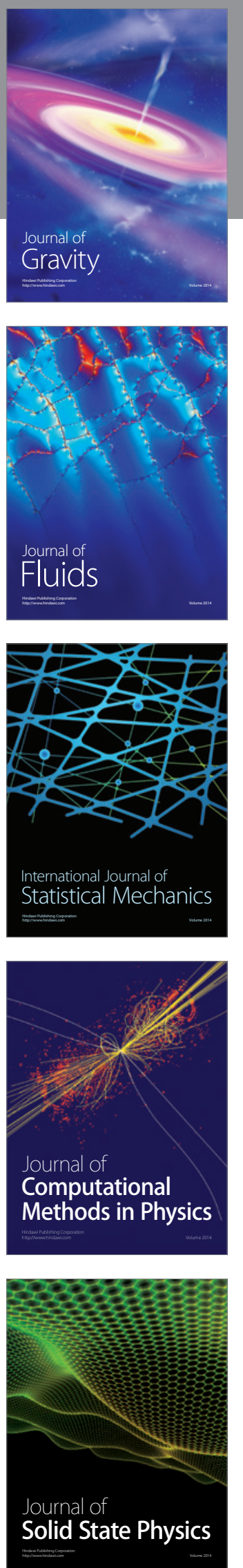

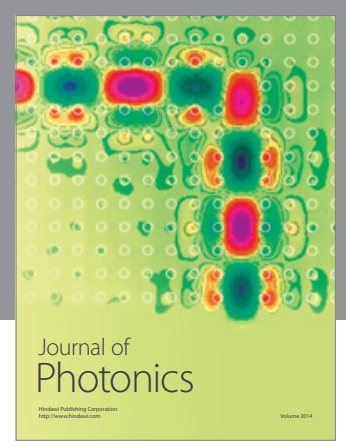

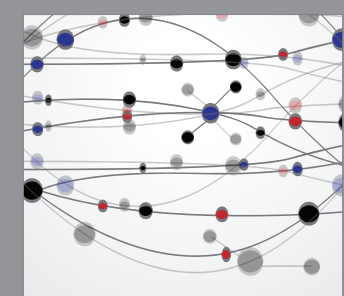

The Scientific World Journal
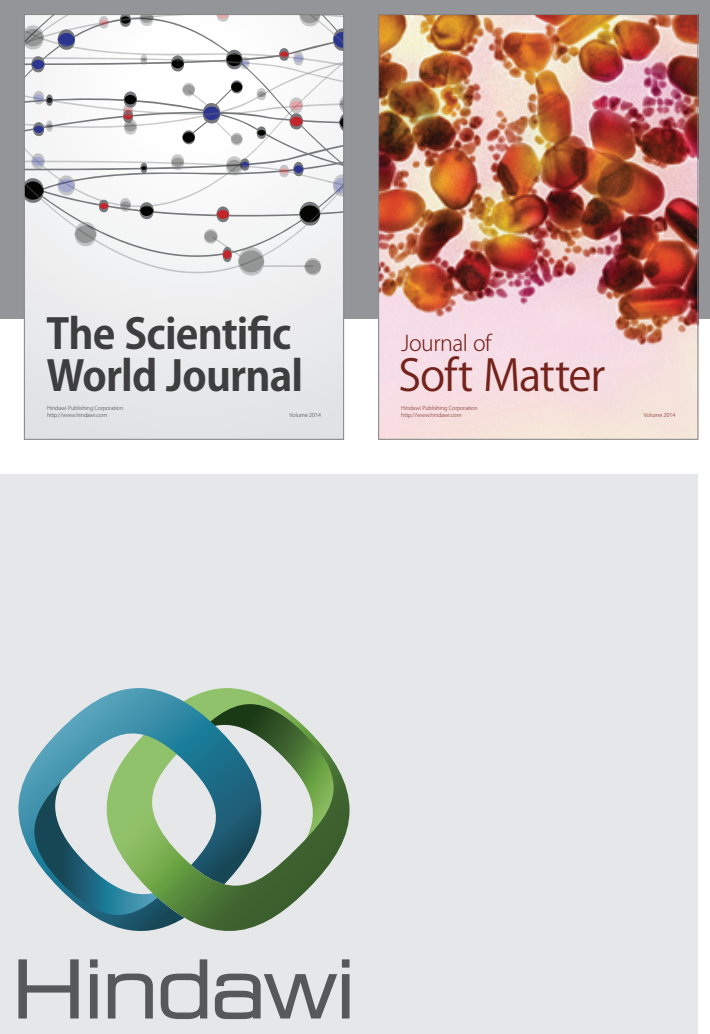

Submit your manuscripts at

http://www.hindawi.com
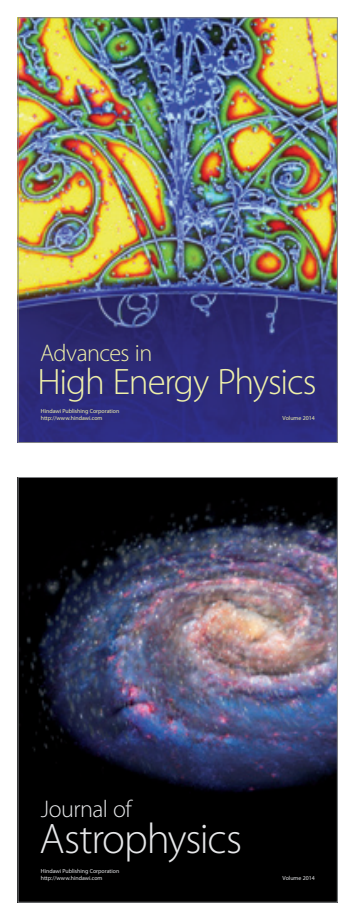
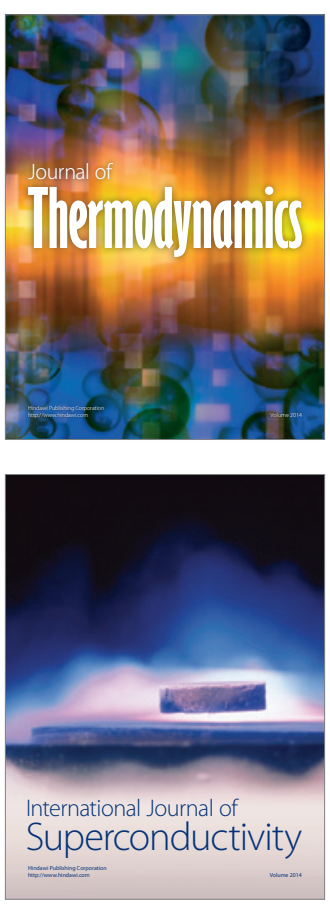
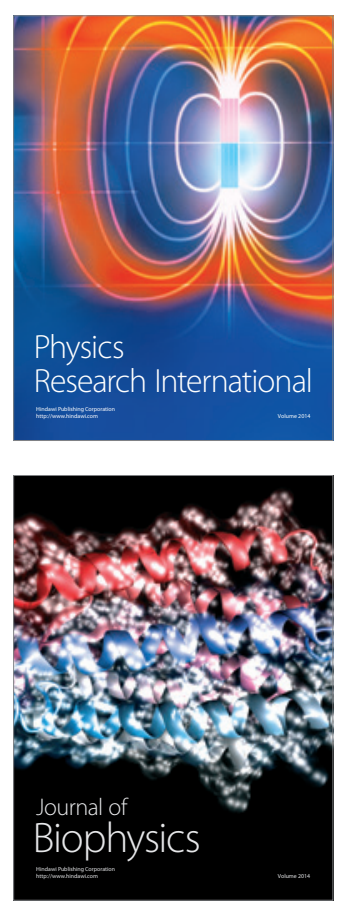
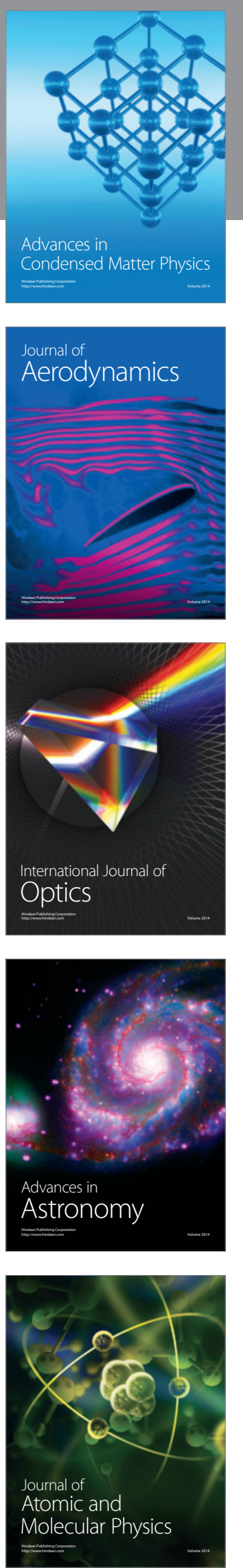